CORRECTION

Series B

\title{
Correction to: On the pervasiveness of difference-convexity in optimization and statistics
}

\author{
Maher Nouiehed $^{1} \cdot$ Jong-Shi Pang $^{1} \cdot$ Meisam Razaviyayn $^{1}$ \\ Published online: 7 March 2019 \\ (c) Springer-Verlag GmbH Germany, part of Springer Nature and Mathematical Optimization Society 2019
}

\section{Correction to: Math. Program. https://doi.org/10.1007/s10107-018-1286-0}

After the publication of the DOI version of [4], the authors were alerted to two examples in the literature that show the value function of, respectively, a constraint-only [2], or an objective-only [3] perturbed (nonconvex) quadratic program (QP) may not be piecewise linear-quadratic, which forms a subclass of the class of piecewise quadratic functions. The latter class of piecewise functions is the principal conclusion of Part (c) of Proposition 9 in the paper [4]. These examples do not contradict the proof of the proposition but invalidate the statement of this part of the proposition. Below we present the correct statement of this part without repeating its proof. We use the same notations as in [4] throughout this erratum; in particular, "dc" stands for differenceof-convex.

Corrected Part (c) of Proposition 9 in [4]. Suppose that $Q$ is copositive on $D_{\infty}$. It holds that

(c) there exist a finite family $\mathcal{F} \triangleq\left\{S_{F}\right\}$ of polyhedra in $\mathbb{R}^{m+k}$ and finitely many quadratic functions $\left\{\mathrm{qp}_{F}\right\}$ such that $\mathrm{qp}_{\mathrm{opt}}(q, b)=\min _{F:(q, b) \in S_{F}} \mathrm{qp}_{F}(q, b)$; hence $\mathrm{qp}_{\mathrm{opt}}(q, b)$ is a piecewise quadratic function on $\operatorname{dom}(Q, D)$.

We should mention that the reference [1, pages 234-238] contains a fair amount of analysis of the value function $\mathrm{qp}_{\mathrm{opt}}(q, b)$, albeit for a fixed pair $(q, b)$ only. In

The original article can be found online at https://doi.org/10.1007/s10107-018-1286-0.

Meisam Razaviyayn

razaviya@usc.edu

Maher Nouiehed

nouiehed@usc.edu

Jong-Shi Pang

jongship@usc.edu

1 Daniel J. Epstein Department of Industrial and Systems Engineering, University of Southern California, Los Angeles, CA 90089-0193, USA 
particular, [1, Theorem 3.129] is related to Parts (c) and (d) of [4, Proposition 9] for a fixed $(q, b)$.

Another oversight in [4] is in the statement of Theorem 12. To apply Proposition 11, we need the value function $\mathrm{qp}_{\text {opt }}(q, b)$ to be quadratic on each polyhedral piece $S_{F}$ in the family $\mathcal{F}$ in Part (c) of Proposition 9. While this is true when $Q$ is positive semidefinite, the statement of the theorem is incomplete without the required quadratic (as opposed to the min of quadratic) property. Below we present the correct statement of this theorem.

Corrected Theorem 12 in [4]. Suppose that $Q$ is copositive on $D_{\infty}$ and that $\operatorname{dom}(Q, D)$ is convex. Then the value function $\mathrm{qp}_{\mathrm{opt}}(q, b)$ is $\operatorname{dc}$ on $\operatorname{dom}(Q, D)$, provided that $\mathrm{qp}_{\text {opt }}$ is a quadratic function on each polyhedral member in the family $\mathcal{F}$ in the above corrected Part (c) of Proposition 9 in [4]. In particular, $\mathrm{qp}_{\mathrm{opt}}(q, b)$ is dc on $\operatorname{dom}(Q, D)$, if $Q$ is positive semidefinite.

We should point out that while not satisfying the quadratic condition in the above theorem, the two numerical examples in [2,3] can be verified to be dc, by the mixing property (Corollary 10 in [4]). For the one in [3] the domain of the value function is $\mathbb{R}^{2}$; for the other one in [2], the domain of the function, which is restricted to the (closed) fourth quadrant in $\mathbb{R}^{2}$ in the reference, can be easily extended to an open convex set containing this quadrant.

Acknowledgements The authors are grateful to Dr. Ying Cui for bringing the reference [3] and Example 4.1 therein to our attention.

\section{References}

1. Bonnans, J.F., Shapiro, A.: Perturbation Analysis of Optimization Problems. Springer, New York (2000)

2. Klatte, D.: On the Lipschitz behavior of optimal solutions in parametric problems of quadratic optimization and linear complementarity. Optimization 16, 819-831 (1985)

3. Lee, G.M., Tam, N.N., Yen, N.D.: On the optimal value function of a linearly perturbed quadratic program. J. Glob. Optim. 32, 119-134 (2005)

4. Nouiehed, M., Pang, J.S., Razaviyayn, M.: On the pervasiveness of difference-convexity in optimization and statistics. Math. Program. Ser. B (2018). https://doi.org/10.1007/s10107-018-1286-0

Publisher's Note Springer Nature remains neutral with regard to jurisdictional claims in published maps and institutional affiliations. 